\title{
CRESCIMENTO E ESTADO NUTRICIONAL DE PRÉ-ESCOLARES RESIDENTES NA REGIÃO AMAZÔNICA DO PERU ${ }^{1}$
}

VELASQUEZ, G; BENAVENTE, L.; CASANOVA, W. Crescimento e Estado Nutricional de Pré-Escolares Residentes na Região Amazônica do Peru. Rev. Bras. Cresc. Des. Hum., São Paulo, III(2), 1993.

Resumo: Foi estudado o estado nutricional de 1684 pré-escolares menores de 5 anos (862 meninos e 822 meninas), residentes na Região Amazônica do Peru; Os dados foram obtidos da Pesquisa Nacional de Nutrição e Saúde (ENNSA), realizada no Peru, em 1984.

$\mathrm{O}$ crescimento e estado nutricional foi avaliado através dos seguintes indicadores: peso para idade, altura para idade, peso para altura e perímetro braquial.

Os reswltados mostram uma elevada prevalência de baixa estatura (porcentagem de adequação à mediana menor que 95\% para o indice altura para idade) e baixa prevalência de inadequação de peso (porcentagem de adequação à média menor que $80 \%$ para o indice peso para altura). De acordo com o indice peso para idade, $38 \%$ das crianças estiveram adequadamente nutridas e apenas 0,8\% apresentaram desnutrição grave.

Palavras-chave: Peru, Amazônia, antropometria, estado nutricional, crianças pré-escolares.

Summary: The growth and nutritional status of 1684 preschol children aged under five years (862 boys and 822 girls), living in the low Peruvian Amazon basin, were studied. The data was obtained from at the health and Nutrition Peruvian National Survey (ENNSA), carried out in 1984.

The nutritional status was assessed by four means-weight for age, height for age, weight for height and arm circunference (AC).

The results show high prevalence of stunting (less than 95\% height for age), and low prevalence of wasting (less than $80 \%$ of weight for height). As judged by weight for age $38 \%$ of children were adequately nourished and only $0,8 \%$ were severe malnutrition.

Key words: Peru, Amazon Basin, anthropometry, nutritional status, preschool children.

1 Trabalho financiado pelo Instituto de Pesquisa da Universidad Nacional de la Amazonia Peruana.

2 Professor associado da Facultad de Medicina - Universidad Nacional de la Amazonia Peruana Iquitos - Peru; doutorando da Faculdade de Saúde Pública da USP - End.: Rua Angelo Menocello, 149 - São Paulo - SP - CEP 05327-070.

3 Professor da Universidad Peruana Cayetano Heredia.

4 Professora da Facultad de Medicina - Universidad Nacional de la Amazonia Peruana. 


\section{INTRODUÇÃO}

O peso inadequado e a baixa estatura são reconhecidos como indicadores de crescimento e desnutrição em pré-escolares. Podem ser aferidos através de índices antropométricos tais como peso para altura (peso/altura) e altura para idade (altura/idade). Esses índices por sua relativa facilidade de aplicação são recomendados pela Organização Mundial da Saúde ${ }^{21,22}$ como instrumentos básicos para o diagnóstico da desnutrição energético-proteica numa comunidade ou num país.

O índice peso/altura relaciona a massa corporal com a altura e é considerado um indicador de desnutrição aguda. O indice altura/idade mede o crescimento linear. Baixas adequações deste indicador caracterizam a desnutrição crônica ou retardo no crescimento. ${ }^{22}$

Os índices antropométricos são comparados com aqueles obtidos de uma população de referência formada por indivíduos em bom estado de saúde e nutrição.

Numerosos inquéritos tanto transversais quanto longitudinais têm sido realizados utilizando essa nnetodologia com o propósito de medir a prevalência a gravidade e a distribuição da desnutrição energético-proteica. Esses estudos são fundamentais na identificação de populações prioritárias para o estabelecimento de programas de desenvolvimento uma vez que o crescimento infantil é um indicador sensível das condições gerais de vida (saúde, moradia, alimentação, saneamento etc.). ${ }^{8,11}$

Em 1984 foi realizado no Peru o segundo inquérito antropométrico de crianças até 72 meses de idade a nivel nacional (Encuesta Nacional de Nutricion y Salud ENNSA). Este inquérito foi realizado pelo Instituto Nacional de Estatística (INE) que colocou os dados à disposição das instituições interessadas na análise dos seus resultados. ${ }^{10}$

O presente trabalho avalia o crescimento e o estado nutricional da população infantil da Região Amazônica Baixa (considerada baixa até 400 m de altitude) a mais extensa e menos povoada do território peruano constituída principalmente por populações dispersas provenicntcs de diversas etnias.

\section{MATERIAL E MÉTODOS}

Os dados deste trabalho foram obtidas da Pesquisa Nacional de Nutrição e Saúde (ENNSA) conduzida no Peru em 1984 pelo instituto Nacional de Estatística (INE) e Ministério da Saúde (MS) com o apoio da International Agency for Development (IAD-USA). ${ }^{10}$
A amostra foi constituida por rcsidências das árcas urbana e rural do País. A informação foi atualizada a partir do "Ccnso Nacional dc Población e Vivienda”, de 1981. O delineamento amostral permite estimativas confiáveis para cada uma das regiões geográficas. A amostra foi probabilística e estratificada, já que as unidades amostrais foram sclecionadas aleatoriamente e a população dividida em cxtratos. ${ }^{10}$

Foram tomadas medidas antropométricas de todas as crianças da amostra, com idade de ate 72 meses. A mensuração de peso e altura foi realizada por equipes de antropomctristas treinados e supcrvisionados por funcionárias do INE, do IAD e do Center for Discase Control (CDC-USA). A verificação das medidas em uma sub-amostra, executadas com instrumentos calibrados, permitiu demonstrar que as variações encontradas entre as regiões não foram devidas a diferentes técnicas de medidas.

Foram seguidas as recomendacões da Organização Mundial da Saúde, utilizando-se, para a tomada de peso, balanças Saltcr (com precisão até $100 \mathrm{~g}$ ). As crianças foram pesadas descalças com o mínimo possível de roupas.

Para a tomada da altura (comprimento, em crianças menorcs de 24 meses), utilizou-se uma régua antropométrica de madeira, com a criança em posição reta e ambas as pernas c pés juntos, com as mãos nos quadris e com a cabeça mantida no plano de Frankfurt (linha imaginária que vai desde o bordo da orbital esquerda à margem superior do meato auditivo externo). A precisão foi de até décimos de centímetro.

As medidas de peso e altura das crianças foram comparadas com os respectivos padrões de peso e altura do "National Centcr for Health Statistics” (NCHS . Os parâmetros desse inquérito são os adotados pela Organização Mundial da Saúde ${ }^{11}$ como populaçao dc referência ou padrão de normalidade.

Os índices peso/idade, pcso/altura e altura/idade foram cxpressos em porcentagens de adequação à mediana da população de rcfcrência ou em "scores z" (número dc unidades, em desvio padrão, que separa a medida observada da esperadas.

Para a avaliação nutricional construiu-se um sub-conjunto do arquivo geral, em formato DBASE III plus, o qual continha informações relevantes para cada criança de até 72 meses de idade, residente na Selva Baixa Peruana.

Foram apenas incluídas crianças com dados antropométricos completos e excludas do estudo 30 crianças com valores " $z$ ” extremamente altos ou baixos; peso/idade $(-6<\mathrm{Z}>+6)$; peso/ altura $(-4<\mathrm{Z}>+6)$; e altura/idade $(-6<\mathrm{Z}>+6)$. 
A prevalência da desnutrição foi estimada usando a classificação de $\mathrm{GOMEZ}^{4}$, que usa o indice pcso/idade (mais de 90\%: normal; 75-90\%: $\mathrm{I}^{\circ}$ grau de desnutrição; 60-74 \%: II ${ }^{\circ}$ grau de desnutrição e, menos de $60 \%$ : II $1^{\circ}$ grau de desnutrição) e a classificação de WATERLOW ${ }^{19,20}$ que usa os índices pcso/altura (mais de 80\%: nonnal; 7080\%: dcsnutriçao moderada; menos de $70 \%$ : desnutrição grave) c altura/idade (mais de 95\%: normal; 90-95\%: I ${ }^{\circ}$ grau de desnutrição; 85-89\%: II ${ }^{\circ}$ grau de desnutrição e menos de 85\%: desnutrição grave)

O processamento foi realizado através do programa Dbase III e a análise estatística foi feita usando-se pacote Epiinto, versão 5.

\section{RESULTADOS}

A distribuição das porcentagens dos índices antropométricos (expressos em porcentagens de adequação à mediana da população de referência) peso/idade, peso/altura e altura/idade, são apresentadas nas tabelas 1, 2, 3 e 4, buscando mostrar o tipo dc desnutrição prevalcnte.

TABELA 1. CLASSIFICAÇÃO DO ESTADO NUTRICIONAL DAS CRIANÇAS, SEGUNDO INDICE: PESO PARA IDADE. REGIÃO AMAZÔNICA, PERU, 1984.

\begin{tabular}{ccc}
\hline ADEQUAÇÃO A MEDIANA & $\mathrm{n}$ & $\%$ \\
\hline$>95$ & 638 & 37,88 \\
$75-95$ & 863 & 51,28 \\
$60-74$ & 170 & 10,10 \\
$<60$ & 13 & 0,77 \\
\hline TOTAL & 1684 & 100,00 \\
\hline
\end{tabular}

Segundo o índice pcso/idade, vcri ; cou-se que apenas 3 ,8 \% das crianças apresentaram-se eutróficas (Tabela 1). A desnutrição de $I^{\circ}$ grau (adequações ponderais situadas entre 75-90 \%) e II $^{\circ}$ grau (adequações entre 60-74\%) somam 61,4 $\%$ e a de III $^{\circ}$ grau (adequação < 60\%) apenas $0,8 \%$.

TABELA 2. PREVALENNCIA (\%) DE DESNUTRIÇAO DE CRIANÇAS MENORES DE 6ANOS, SEGUNDO IDADE (CLASSIFICADO GOMEZ). REGIÃO AMAZÔNICA, PERU, 1984.

\begin{tabular}{ccccc}
\hline $\begin{array}{c}\text { idades } \\
\text { meses }\end{array}$ & $\mathrm{n}$ & $\begin{array}{c}\text { Desnutrição } \\
1^{\circ} \text { grau }\end{array}$ & $\begin{array}{c}\text { Desnutrição } \\
1 \mathrm{I}^{\circ} \text { e } 111^{\circ} \text { grau }\end{array}$ & Total \\
\hline $0-5$ & 155 & 30,3 & 5,2 & 35,5 \\
$6-11$ & 153 & 45,1 & 13,7 & 58,8 \\
$12-23$ & 278 & 51,4 & 19,8 & 71,2 \\
$24-72$ & 1098 & 55,1 & 9,0 & 64,1 \\
\hline
\end{tabular}

Nos seis primeiros meses de vida a prevalência total de desnutrição já é alta (35,5\%), como pode ser observado na tabela 2 , sendo que a de $\mathrm{II}^{\circ}$ grau e $\mathrm{III}^{\circ}$ grau, atingem $5 \%$. Pode-se verificar um aumento gradual da prevalência de desnutrição até o segundo ano de vida, das formas de $\mathrm{I}^{\circ}$ grau e as de $\mathrm{II}^{\circ}$ e $\mathrm{III}^{\circ}$ graus (51 e 20\%, respectivamente); a partir daí as taxas não seguem o mesmo padrão.

TABELA 3. CLASSIFICAÇÃO DO ESTADO NUTRICIONAL DAS CRIANÇAS, SEGUNDO INDICE PESO PARA ALTURA. REGIÃO AMAZÔNICA, PERU, 1984.

\begin{tabular}{ccccc}
\hline \% ADEQUAÇÃO & \multicolumn{2}{c}{12 MESES } & \multicolumn{2}{c}{$12-72$ MESES } \\
A MEDIANA & $\%$ & $\mathrm{n}$ & $\mathrm{n}$ & $\%$ \\
\hline$>80$ & 300 & 97,40 & 1370 & 99,56 \\
$70-80$ & 7 & 2,27 & 5 & 0,37 \\
$<70$ & 1 & 0,33 & 1 & 0,07 \\
\hline TOTAL & 308 & 100,00 & 1376 & 100,00 \\
\hline
\end{tabular}

A prevalência de desnutrição aguda grave, aferida através do índice peso/altura, é baixa $(2,6 \%)$ em crianças menores de 12 meses e de $0,44 \%$ entre aquelas de 12 a 72 meses, como pode ser obscrvado na tabela 3 .

Os resultados da tabela 4 ilustram a distribuição do índice altura/idade; verifica-se que 67\% das crianças menores de 72 meses apresentam valores abaixo deste índice.

TABELA 4. CLASSIFICA ÇÃO DO ESTADO NUTRICIONAL DAS CRIANÇAS, SEGUNDO INDICE ALTURA PARA IDADE. REGIÃO AMAZÔNICA, PERU, 1984.

\begin{tabular}{ccc}
\hline $\begin{array}{c}\text { \% ADEQUAÇAO } \\
\text { A MEDIANA }\end{array}$ & $\mathrm{n}$ & $\%$ \\
\hline$>95$ & 564 & 33,49 \\
$90-95$ & 662 & 39,31 \\
$85-89$ & 356 & 21,14 \\
$>85$ & 102 & 6,06 \\
\hline TOTAL & 1684 & 100,00 \\
\hline
\end{tabular}

As Tabelas 5 e 6 mostram que a desnutrição aferida através dos índices peso/altura e altura/idade são independentes do sexo $\left(X^{2}=0,06\right.$, p > 0,05; $X=2.09, p>0,05$ ), porém a prcvalência de baixa estatura esteve associada à idade $\left(X^{2}=210,9 ; p<0,001\right)$. 
TABFLA 5. DISTRIBUIÇÃO DAS CRIANÇAS, SEGUNDO SEXO E IDADE E INIDICE PESO PARA ALTURA. REGIÃO AMAZÔNICA, PERU, 1984.

\begin{tabular}{cccc}
\hline & \multicolumn{3}{c}{ PESO PARA ALTURA } \\
\hline & $80 \%$ & $70-80 \%$ & $70 \%$ \\
\hline SEXO & & & \\
MASCULINO & 858 & 3 & 1 \\
FEMININO & 812 & 9 & 1 \\
\hline TOTAL & 1670 & 12 & 2 \\
\hline IDADE (anos) & & & \\
$>1$ & 300 & 7 & 1 \\
1 & 274 & 4 & 0 \\
2 & 281 & 1 & 0 \\
3 & 296 & 0 & 0 \\
4 & 281 & 0 & 0 \\
5 & 238 & 0 & 1 \\
\hline TOTAL & 1670 & 12 & 2 \\
\hline${ }^{2}=0,06$ NS & & &
\end{tabular}

A associação da estatura à idade pode scr explicada pelo fato de que a proporção de crianças dcsnutridas crônicas (altura/idadc < 95\%), no grupo de menores de 12 meses é, aproximadamente, a metade dos outros grupos etários (Tabela 6).

Uma análise mais detalhada da evolução dos índices antropométricos segundo faixa etária são mostrados na Figura 1.

São apresentadas as médias das adcquacões dos indicas antropomctricos da população estuda-
TABELA 6. DISTRIBUIÇÃO DAS CRIANÇAS, SEGUNDO SEXO E IDADE E INDICE ALTURA PARA IDAI)E. REGIÃO AMAZÔNICA, PERU, 1984.

\begin{tabular}{ccccc}
\hline \multicolumn{5}{c}{ ALTURA PARA IDADE } \\
\hline & $>95 \%$ & $90-95 \%$ & $85-89 \%$ & $85 \%$ \\
\hline SEXO & & & & \\
MASCULINO & 291 & 331 & 190 & 50 \\
FEMININO & 273 & 331 & 166 & 52 \\
\hline TOTAL & 564 & 662 & 356 & 102 \\
\hline IDADE $^{\mathrm{b}}$ (anos) & & & & \\
$<1$ & 209 & 87 & 84 & 74 \\
1 & 87 & 121 & 61 & 9 \\
2 & 84 & 114 & 65 & 10 \\
3 & 74 & 130 & 66 & 26 \\
4 & 62 & 111 & 80 & 28 \\
5 & 48 & 104 & 69 & 18 \\
\hline TOTAL & 564 & 662 & 336 & 102 \\
\hline${ }^{\mathrm{a}} \mathrm{X}^{2}=209 \mathrm{NS},{ }^{\mathrm{b}} \mathrm{X}^{2}=210, \mathrm{p}<0,001$ & &
\end{tabular}

da A partir do segundo semestre de vida observase uma queda progressiva dos trcs indices em relação ao valor teórico esperado (100\%), depois uma estabilização em torno de $90 \%$. Isto ocorre particularmente com os indicas pcso/idade e altura/idade. Tal comportan ento revela o comprometimento sistemático do crescimento infantil nos primeiros meses de vida Diferencia-se deste comportamento o índice peso/altura que volta a ser igual ao padrão de referência depois do segundo ano de vida

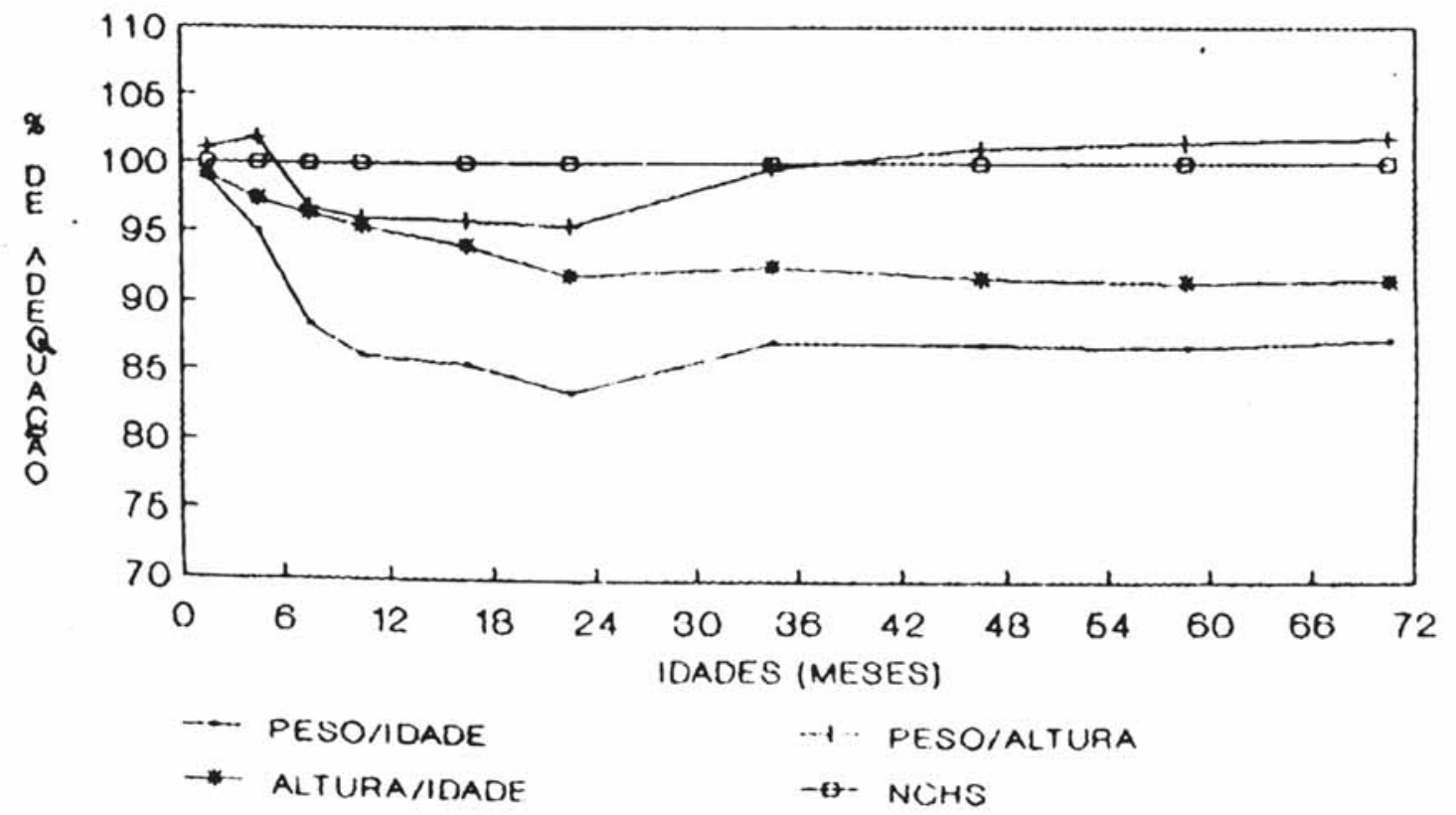

Fiğura 1 - Médias das adequaçōes dos indices peso/idade, peso/altura, altura/idade em relação à população de referência (NCIIS). Crianças de 0 - 6 anos da Região . Imazônica Baixa do Peru, 1984 


\section{DISCUSSÃO}

Neste trabalho foram apresentados os resultados de um inquérito antropométrico transversal realizado no Peru no ano de 1984 com amostragcm nacional da população. O objetivo principal foi o de retratar a situação nutricional e de saúde da infância no Peru. Este inquérito contou com condições adequadas de equipamento e treinamento de pessoal na coleta dos dados.

Os resultados apresentados nesta pesquisa retratam apenas o perfil de crescimento e estado nutricional de crianças menores de 6 anos residentes na Região Amazônica Baixa do Peru.

Para a descrição do crescimento dessa população foi utilizada a população de referência OMS/NCHS. ${ }^{21}$ Para fins práticos assumindo que o potencial de crescinnento das crianças em ambas as populações é semelhante e afetado principalmente pelas condições do meio ambiente $\mathrm{e}^{5,7,11,13,21}$ aceita-se também como padrão de normalidade.

As prevalências de desnutrição de acordo com a classificação de GOMEZ (Tabela l) aprcscntam-sc semelhantes às prevalencias encontradas nas Rcgiões Nortc (Amazônica) e Nordeste do Brasil. ${ }^{9,17}$

A partir da distribuição das adequações dos indicadores peso/altura e altura/idade podemos diferenciar formas agudas e crônicas de desnutrição energético-proteica. Utilizando esses dois índices foi possivcl identificar que nnenos de $3 \%$ das crianças amazônicas aprescntaram desnutrição aguda e uma alta proporção mostrou desnutrição crônica (déficit de altura). Nenhum desses tipos de desnutrição esteve associado ao sexo. Apenas a desnutrição crônica mostrou associação com a idade de forma similar ao achado em outro inquérito antropométrico

Esses resultados podem sugerir algumas explicações. Embora na Região Amazônica a lactância materna seja prolongada, o início da introdução de outros alimentos diferentes do leite é precoce e está constituída, principalmente, por banana e mandioca. Pela maneira como estes alimentos são preparados é possível que diminua sua densidade energética e proteica fazendo com que as crianças se saciem rapidamente mesmo que não satisfaçam seus requerimentos nutricionais. ${ }^{2}$ Uma dieta monóto- na determina provavelmente pouco interesse e apetite nas crianças pequenas.

Existem evidências ${ }^{3}$ que indicam má digestibilidade dos carboidratos da banana, quando esta é consumido antes da maturação completa, prática habitual na Região Amazônica.

Além disso a probabilidade de ingestão de alimentos preparados sem muita higiene é grande, aumentando o risco de infecções. Têm sido amplamente demonstrado o papel das infecções na piora do estado nutricional a partir dos seus efeitos catabólicos inespecificos. ${ }^{15.16}$ Esta situação mantém as crianças em ingestão alimentar marginal de forma crônica sem comprometimento significativo do peso mas com comprometimento do crescimento linear. O retardo no crescimento tem também sido associado com frequentes episódios de diarréia. Outros inquéritos realizados em crianças desta região mostraram alta incidência de diarréias e enteroparastoscs. ${ }^{10,12}$

Recentemente BAGENHOLM e col. ${ }^{1}$ a partir de um estudo com delineamento transversal mostraram que a baixa estatura em crianças lemenitas durante o primeiro ano de vida está associada à depleção da massa muscular e do tecido adiposo.

Por outro lado alguns autores têm enfatizado o papel de possíveis fatores genéticos e adaptativos na etiologia do retardo no crescimento. ${ }^{5}$

Finalmente os mecanismos que levam uma criança à diminuição na velocidade do crescimento ainda não estão bem compreendidos.

Entretanto uma porcentagcm pequena de crianças amazônicas apresentam desnutrição aguda; esse número pode ser considerado baixo dentro de um amplo espectro de prevalências encontradas em outras regiões do mundo ${ }^{6}$ e semclhante às encontradas nos países latino-americanos (dados da OMS citado por MONTEIRO e col. ${ }^{17}$ ) onde esses níveis não alcançam rclevância epidemiológica. ${ }^{6}$

Concluindo o perfil de crescimento das crianças amazônicas mostra duas características bem definidas: as formas crônicas de desnutrição são as mais prevalentes evidenciadas pelo distanciaincnto do indice altura/idadc com relação à população de referência. Por outro lado a desnutrição aguda encontrada apenas em uma pequena proporção de crianças não apresenta relevância epidemiológica. 


\section{REFERÊNCIAS BIBLIOGRAFICAS}

1. BAGENHIOLM, G.; NASHER, A.A.A.; KRISTIANSSON, B. Stuntedand tissue depietion Yemeni children. Eur. J. Clin. Nutr., 41, 425-33, 1990.

2. BENAVENTE, L.; BENAVENTE, M.; CAMPOS,M. Estado nutricional en menores de 6 anos en el Peru. Lima, 1986. [Tesis de Maestria - Facultad de Medicina - Universidad Peruana Cayetano Heredia].

3. ENGLYST, H.; CUMMINES, H. Digestion of the carbohydrates of banana (Musa paradisiaca sapientum) in the human smail intestine. Amer. J. trop. Med. Hyg., 44: 42-50, 1986.

4. GOMEZ, F. et al. Mortality in seeond and third degree malnutrition. J. trop. Pediatr., 22: 7783,1956.

5. GREWLICH, W. A comparison of physical growth and development of American born and native Japanese children. Am. J. Phys. Anthrop., 15: 489-5 1 5, 1957.

6. HAAGA, J.; KENRICK, C.; TEST, K.; MASCON, J. An estimate of the prevalence of child malnutrition in developing countries. World Hlth. Statist. Quart., 38: 331 -33, 1985.

7. HABITCH, J; MARTORELL, R.; YARBROUGH, C.; MOLINA, R.; KLEIN, R. Height and weight standards for preschool children. How relevant are ethnic diferences in growth potential. Lancet, 1: 611-15, 1974.

8. HANSEN, J. D. L.; FREESEMAN, C.; MOODIE, A.; EVANS,D. What does nutritional growth retardation imply? Pediatrics, 47: 299-313, 1971.

9. INSTITUTO NACIONAL DE ALIMENTAÇÃO E NUTRIÇÃO (INAN)/MINISTÈRIO DA SAÚDE. Pesquisa nacional sobre saúde e nutrição - resullados preliminares. Brasilia, 1990.

10.INSTITUTO NACIONAL DE ESTADISTICA Y MINISTERIO DE SALUD. Encuesta nacional de nutrición y salud - informe gencral. Lima, 1984.

11. JORDAN, J. R. Crecimiento del niño como indicador de salud. In Salucl infantily atención primaria en las Américas. Organización Panamericana de la Salud, 1984. (Publicación Cientifica, 461).
12. LUMBRERAS, H; NAQUIRA, C. Parasitismo intestinal en el Peru: encuesta epidemiológica de 12 localidades. In Libro de Resumenes. IV Jornada Cientifica de la Universidad Peruana Cayetano Heredia, Lima, 1986. p. 294.

13. MARTORELI, R; LECHTING A; HABITCH, J.; YARBROUCH, C; KLEIN, R. Normas antropométricas de crecimiento fisico para paises en desarrollo: nacionales o internacionales? Bol. of Sanit. Panam., Washington, 22: 525.29, 1975.

14.MARTORELL, R; YARBROUGH, C.; LECHTING, A; HABITCII, J. P; KLEIN, R. Diarrhocal disease and growth retardation in preschool Guatemalan children. Am Phys. Anthropol., 43: 341-46, 1975.

15. MAIA, I, J; UMBRUTIA, J. J; ALBERTAZZI, C; PELLECER, O; ARELLANO, E. Imfuence of recent infections on nutrition and growh of children in Guatemala. Am. J. Clin. Nutr., 25: 1267.75, 1972.

16. MAIA, I, J; UMBRUTIA, J. J; LECHING, A. Infection and nutrition of child hood of low socioeconomic rural community. Am. J. CIin. Nutr., 24: 249.58, 1971.

17. MONTEIRO, C. A; BENICIO, M. H. A; GUVEIA, N. C. Growth and nutritional status of Brazilian children: findings from the 1999 National Health and Nutrition Survey. Geneve, 1991. (WHO Nutrition Unit. Country Studies on Nutritional Anthropometry).

18. REES, D. G; HENRY, C. J. K; DISKETT, P; SHEARS, P. Measures of nutritional status. Survey of young children in North-East, Brazil. Lancet, 1: 87.9, 1987.

19 WATERLOW, J. C. Classification and definition of protein-calorie malnutrition. Brit. mecl. J., 2: 87.9, 1973.

20. WATERLOW, J. C. Note the assessment and classification of protein energy malnutrition in children. Lancet, 2: 87.9, 1973.

21. WORLD HEALTH ORGANIZATION. Measuring change in nutritional status guide lines for assessing the nutritional impact of supplementary feeding programmes for vulnerable groups. Geneve, 1983.

22. WORLD HEALTH ORGANIZATION WORKING GROUP. Use and interpretation of anthropometric indicators of nutritional status. Bull. Wld. Hlth, Org., 64: 929.41, 1986. 